\title{
ÉPIDÉMIOLOGIE DE LA TRICHINELLOSE EN ITALIE : CORRELATION ENTRE LE CYCLE SAUVAGE ET L'HOMME
}

\author{
E. POZIO, P. Rossi, M. AMATI
}

\begin{abstract}
RÉSUMÉ. En Italie, la trichinellose est une zoonose causée par Trichinella nelsoni, qui présente un cycle exclusivement sauvage, concernant les animaux sauvages et synanthropiques (renards, loups, chiens errants, sangliers, porcs élevés à l'état demi-sauvage). La récente diffusion et le développement des populations de sangliers sauvages dans toute l'Italie, ont causé deux foyers épidémiques de trichinellose humaine en 1985 et 1986, concernant environ cent personnes. L'habitude de consommer de la viande de renard a causé aussi d'autres infections humaines. Les résultats des examens sérologiques de populations sauvages de sangliers ont montré l'importance du rôle joué par ce porcin dans l'épidémiologie de la maladie. L'examen des populations de rongeurs a mis en évidence l'infection chez ces animaux (rat, porc-épic). Des corrélations épidémiologiques entre le cycle sauvage et l'homme sont établies.
\end{abstract}

Mots-clés : Trichinella. Épidémiologie. Italie. Cycle sauvage. Infections humaines.

\section{Epidemiology of trichinellosis in Italy: correlation between the wild cycle and man.}

SUMMARY. In Italy trichinellosis is a zoonosis caused by Trichinella nelsoni, with an exclusively sylvatic cycle involving wild and synanthropic animals (fox, wolf, stray dog, wild boar, foraging pig). The recent increase and diffusion of wild boar population in all Italy, have caused two human epidemic foci of trichinellosis involving about 100 persons in the years 1985-1986. The custom of eating fox meat have caused other human infections. The results of serological examinations of wild boar population showed the role played by this animal in the epidemiology of the disease. The examination of rodent population has pointed out the infection in these mammals (rat, porcupine). The epidemiological implications of wild cycle on man are reported.

Key-words : Trichinella. Epidemiology. Italy. Wild cycle. Human infections.

\section{Introduction}

En Italie, la trichinellose est une parasitose endémique, concernant principalement les animaux sauvages et synanthropiques. Parmi les animaux sauvages, le renard (Vulpes vulpes) a été l'objet de nombreuses enquêtes, qui ont conduit à des résultats de diagnostic positifs dans presque toute l'Italie, à l'exception de la Sicile et de la Sardaigne (Mantovani et coll., 1980). Le loup (Canis lupus) a été

Laboratorio di Parassitologia, Istituto Superiore di Sanità, Viale Regina Elena, 299 00161 Rome, Italie.

Accepté le 6 décembre 1986. 
trouvé parasité en Italie du Sud, dans six cas sur six (Corcione et Musacchio, 1966; Colella, 1975). On a signalé le parasite aussi chez la fouine (Martes foina) (De Filippis et Nardi, 1968) et le blaireau (Meles meles) (Orlandi, 1972). Des enquêtes menées sur les chiens errants et sauvages ont montré des taux d'infestation atteignant $24 \%$ dans le Gargano (Pouilles, Italie du Sud) (Nardi, 1960). Au contraire, les enquêtes réalisées sur les rongeurs n'ont pas permis de déceler le parasite, à l'exception de quelques rongeurs trouvés positifs dans deux foyers de trichinellose humaine en Sicile dans les années précédant 1945.

En 1958, l'examen trichinelloscopique de la viande de porc est devenu obligatoire en Italie et, depuis, 4 animaux seulement ont été découverts positifs. De 1900 à 1978, on a signalé 4 cas isolés (à la suite de consommation de viande importée) et 12 foyers épidémiques de trichinellose, dont un causé par de la viande de sanglier (Mantovani et coll., 1980).

De 1976 à 1984, l'Annuario di Statistica Sanitaria (1976-1984) a signalé 48 cas, pour lesquels on manque toutefois de données épidémiologiques et cliniques. Nous ne traiterons pas dans ce travail des foyers humains causés par de la viande de cheval importée de l'étranger en 1975, 1984 et 1986 (Mantovani et coll., 1980; Parravicini et coll., 1986; Pozio et coll., en préparation).

Ce travail présente les résultats des enquêtes menées : 1) à la suite des nouveaux foyers de trichinellose humaine signalés en Italie du Sud; 2) dans les zones reconnues endémiques pour le cycle sauvage. Ces enquêtes ont concerné l'homme, les animaux domestiques, synanthropiques et sauvages.

\section{Matériel et méthodes}

Homme : tous les sujets qui présentaient les signes cliniques distinctifs de la parasitose ont été soumis à l'examen sérologique par le test de l'immunofluorescence indirecte (IF).

Animaux domestiques et d'élevage : à l'occasion des foyers humains, nous avons examiné par le test IF les animaux domestiques présents dans les zones intéressées par l'épidémie, aussi bien que des chiens errants abattus auprès du Chenil Municipal de Gravina, dans les Pouilles (Province de Bari, Italie du Sud).

Sanglier sauvage : nous avons pu examiner par le test IF des sérums de sangliers sauvages en provenance du Parc Régional des "Monti dell'Uccellina " (Province de Grosseto, Italie Centrale).

Rongeurs : nous avons capturé les rats par des pièges "Tomahawk " (live trap mod n. 104), et les souris et les insectivores par des pièges "Longworth " live trap. Nous avons utilisé du beurre d'arachide comme appât. En outre, un porc-épic (Hystrix cristala) a été capturé par des chasseurs.

Examen trichinelloscopique : nous avons fait l'examen trichinelloscopique du diaphragme et du muscle masséter des rongeurs et des insectivores. 
Digestion pepsique arlificielle : chez tous les rongeurs nous avons effectué la digestion artificielle d'environ $70 \%$ des muscles. Cette méthode a été employée aussi chez les renards tués dans la province de Rome (Corselli et coll., 1983) et chez un spécimen provenant de la province de Matera (Italie du Sud), chez un porc-épic et un chat domestique.

Xénodiagnose : nous avons nourri des souris Swiss et/ou des rats Wistar avec les carcasses des rongeurs, aussi bien pour mettre en évidence l'infestation que pour isoler le parasite.

Immunofluorescence indirecte (IF) : nous avons employé la technique de Sulzer (1965), en utilisant comme antigène les larves de la souche isolée chez un renard de la province de Teramo (Italie Centrale). Nous avons effectué un test préliminaire d'immunoélectrophorèse, qui a donné une réaction très positive entre le sérum anti-souris et le sérum de Apodemus et Clethrionomys. Pour la routine, nous avons testé des sérums dilués au $1 / 20^{\mathrm{e}}-1 / 40^{\mathrm{e}}$. Le test a été répété avec des dilutions croissantes jusqu'à l'extinction de la valeur de positivité.

Typage enzymatique : toutes les souches du parasite obtenues pendant notre travail, sont maintenues sur souris Swiss et/ou rat Wistar, ce qui nous a permis de les identifier par la méthode des isoenzymes (électrophorèse sur gel d'amidon pour 26 systèmes gène-enzyme) (Pozio et coll., 1987).

\section{Résultats}

Dans les 3 dernières années, 2 foyers d'infestation ont été signalés en Italie du Sud.

En décembre 1985, à Gravina, dans les Pouilles, 80 personnes, ayant consommé des saucisses crues de sanglier, ont présenté des symptômes de trichinellose. 67 d'entre elles, chez lesquelles on a pu effectuer un prélèvement sanguin, se sont avérées sérologiquement positives. Aucun sujet n'a été hospitalisé, la symptomatologie étant assez légère. Le sanglier infecté provenait d'un élevage à caractère familial et n'avait pas été soumis à l'examen trichinelloscopique. Nous avons examiné 22 sangliers provenant du mème élevage, tous négatifs. 4 chiens et 2 chats qui avaient consommé des déchets de l'abattage du sanglier parasité, ont donné un résultat sérologiquement positif. Nous avons pu isoler le parasite d'un des 2 chats. Nous avons aussi effectué l'examen parasitologique et sérologique de 38 Rattus rattus et pu isoler le parasite de l'un d'entre eux, trouvé positif (voir tableau I). En cette même année, à Cosenza (Italie du Sud), 2 sujets, qui avaient consommé de la viande de renard, ont été hospitalisés, et se sont avérés parasitologiquement positifs pour Trichinella.

En avril 1986, le deuxième foyer humain a été signalé à Irsina (province de Matera, Italie du Sud), village situé à quelques dizaines de kilomètres de Gravina, dans les Pouilles, où avait été trouvé le premier foyer. 20 sujets, ayant consommé de la charcuterie contenant de la viande d'un sanglier élevé dans une ferme, ont 
présenté de légers symptômes et se sont avérés positifs sérologiquement. Aucun sujet n'a été hospitalisé. Nous avons effectué l'examen sérologique de 3 porcs, 2 sangliers, 1 chat et 4 chiens domestiques provenant de la même ferme ; un porc seulement s'est révélé positif sérologiquement. Nous avons aussi effectué l'examen parasitologique et sérologique de 5 Rattus rattus, avec des résultats négatifs (Tableau $I$ ).

Nous avons pu examiner par le test IF, 77 sérums de Sus scrofa, provenant des "Monti dell'Uccellina ", dont $11(14 \%)$ ont donné un résultat positif (voir tableau $I$ ).

Quant aux Rongeurs, un Hystrix cristata a été reconnu positif à l'examen parasitologique. Six Rattus norvegicus sur 80 examinés, provenant de la province de Teramo, ont également été reconnus positifs parasitologiquement et sérologiquement, ce qui a permis d'isoler 5 souches de ce parasite (voir tableau I). Tous les autres rongeurs (228) et insectivores (8) ont donné un résultat négatif aux examens parasitologiques et sérologiques (voir tableau $I$ ). Les souches obtenues d'un chat, d'un $R$. rallus et des $6 R$. norvegicus, ont été identifiées par la méthode des isoenzymes (Pozio et coll., 1987), comme appartenant toutes à l'espèce $T$. nelsoni.

Tableau I. - Animaux examinés en 1985-1986 en Italie Centrale et du Sud.

\begin{tabular}{|c|c|c|c|c|c|}
\hline \multirow[t]{2}{*}{ Espèces } & \multicolumn{4}{|c|}{ Examens exécutés ${ }^{1}$} & \multirow{2}{*}{$\begin{array}{l}\text { Souches } \\
\text { obtenues }\end{array}$} \\
\hline & $\mathrm{ET}^{2}$ & $\mathrm{DA}^{3}$ & $\mathrm{X}^{4}$ & $\mathrm{IF}^{\mathbf{j}}$ & \\
\hline Porc domestique & _- & - & 一 & $1 / 3$ & - \\
\hline Chat domestique & $1 / 1$ & $1 / 1$ & $1 / 1$ & $2 / 3$ & 1 \\
\hline Chien domestique & - & - & - & $4 / 11$ & -- \\
\hline Chien errant & - & - & - & $0 / 35$ & - \\
\hline Sanglier d'élevage & - & - & - & $0 / 24$ & - \\
\hline Sanglier sauvage & - & - & - & $11 / 77$ & - \\
\hline Hystrix cristata & $0 / 1$ & $1 / 1$ & - & - & - \\
\hline Rattus norvegicus & $4 / 80$ & $6 / 80$ & $5 / 80$ & $6 / 80$ & 5 \\
\hline Rattus ratlus & $1 / 100$ & $1 / 100$ & $1 / 100$ & $1 / 100$ & 1 \\
\hline Apodemus sylvaticus & $0 / 102$ & $0 / 102$ & $0 / 102$ & $0 / 102$ & - \\
\hline A podemus flavicollis & $0 / 28$ & $0 / 28$ & $0 / 28$ & $0 / 28$ & 一 \\
\hline Clethrionomys glareolus & $0 / 26$ & $0 / 26$ & $0 / 26$ & $0 / 26$ & - \\
\hline Mus musculus & $0 / 15$ & $0 / 15$ & $0 / 15$ & $0 / 15$ & 一 \\
\hline Crocidura suaveolens & $0 / 8$ & $0 / 8$ & $0 / 8$ & $0 / 8$ & - \\
\hline
\end{tabular}

1. $\mathrm{N}$ positifs $/ \mathrm{N}$ examinés. 2. $\mathrm{ET}=$ examen trichinelloscopique. $3 . \mathrm{DA}=$ digestion artificielle. 4. $\mathrm{X}=$ xénodiagnose. 5 . IF $=$ immunofluorescence indirecte.

\section{Discussion}

Déjà en 1958, en province de Matera, Lo Nigro signalait un cas de trichinellose humaine due à la consommation de viande de sanglier sauvage. Par la suite, un 
foyer de 6 cas humains a été signalé dans la même zone par le même auteur (Lo Nigro et coll., 1978).

Les foyers d'infection humaine de 1985 et 1986, liés à la consommation de viande de sanglier, mettent en évidence l'importance du rôle joué par ce porcin dans l'épidémiologie de la trichinellose en Italie du Sud.

Actuellement, on assiste d'une part à la diffusion croissante des populations sauvages de sangliers, cause, dans certaines zones de dommages importants pour les cultures et la faune, d'autre part au développement de l'élevage de ce suidé souvent croisé avec le porc.

L'introduction, pour la chasse, de races de sanglier en provenance de l'Europe de l'Est, plus prolifiques par rapport à la race originaire italienne (9-10 petits contre 1-2 petits par femelle par an), constitue une des causes principales ayant déterminé la situation actuelle.

Dans les dernières années, de nombreuses zones de chasse ont été transformées en parcs nationaux, où la faune est protégée. L'énorme développement des populations sauvages de sangliers qui en a résulté a causé des phénomènes aberrants, tel le cannibalisme, dont l'importance dans l'épidémiologie de la trichinellose est évidente.

En outre, la présence, dans toute l'Italie Centrale et du Sud, de décharges d'ordures sans enceinte et de déchets abandonnés un peu partout, facilite la rencontre des populations sauvages de ce suidé avec les animaux synanthropiques, tels rats, renards, chiens et chats.

D'autre part, l'élevage du sanglier, qui est en train de se répandre de plus en plus, se fait la plupart du temps à l'état demi-sauvage, souvent en zones boisées. En plus, les carcasses de renards et de chiens errants abattus sont souvent utilisées pour nourrir les sangliers d'élevage. L'abattage est pratiqué à la ferme, donc en dehors de tout contrôle vétérinaire.

Dans les deux foyers de 1985 et 1986, la légèreté des symptômes de $T$. nelsoni montrés par les sujets atteints avait laissé soupçonner, tout au début, une intoxication alimentaire non spécifique. Nos observations ne concordent pas avec celies de Ozeretskovskaya (1978) qui avait remarqué que, chez l'homme, les symptômes dus à $T$. spiralis sont plus légers que ceux dus à $T$. nelsoni, après ingestion de la même quantité de larves. Certains sujets, plus sensibles, ont manifesté des signes cliniques plus accentués. Fort probablement, ces épisodes épidémiques, liés au manque de contrôles vétérinaires des animaux abattus à domicile, ou du gibier, et à l'habitude de consommer de la charcuterie fraîchement préparée, sont plus fréquents qu'on ne le pense et passent inaperçus, étant confondus avec des intoxications alimentaires non spécifiques. Seul un médecin attentif et expert peut soupçonner la cause réelle de l'infection; tel a été le cas des deux derniers foyers. En début d'infection, les ophtalmologistes surtout sont concernés, les premiers symptômes consistant en œdème des paupières et en uvéite.

Quant au renard, on peut le considérer comme témoin de l'infection en milieu sauvage et péridomestique.

Les recherches effectuées par l'Institut de Parasitologie de l'Université de 
Rome, de 1981 à 1983 (Corselli et coll., 1983), ont révélé 11 renards positifs à l'examen parasitologique sur 1134 examinés en provenance de la province de Rome. Par ailleurs, à l'Institut Zooprophylactique de Teramo, de 1982 à 1985, sur 672 renards examinés, 4 ont été trouvés parasités (Istituto Zooprofilattico dell'Abruzzo e del Molise, 1982-1985). A la section Matera du même Institut, 4 renards sur 40 ont été reconnus positifs à l'examen parasitologique (Santagada, comm. pers.).

Ces huit dernières souches vulpines isolées ont été identifiées par la méthode des isoenzymes, comme appartenant à l'espèce T. nelsoni (Pozio et coll., 1987).

Les cas d'infection humaine causés par l'ingestion de viande de renard sont signalés sporadiquement en Italie. En 1961, en Italie du Nord (province de Trento), un foyer de trichinellose humaine a été signalé, concernant 9 personnes ayant consommé de la charcuterie crue préparée avec de la viande de renard (Pampiglione et Doglioni, 1971).

Néanmoins, la présence de ce parasite chez des rongeurs (Pozio, 1986) montre qu'ils jouent aussi un rôle assez important dans l'épidémiologie de cette parasitose.

Remeraiements. Nous remercions très vivement Mme F. Mancini Barbieri pour l'assistance technique fournie.

\section{BIBLIOGRAPHIE}

AnNuahio di Statistica SANitaria : 1976, 1977, 1978, 1979, 1980, 1981, 1982, 1983, 1984.

Colella G. : Indagini sulla trichinosi in provincia di Matera: prima segnalazione nel cane. Vet. ital., $1975,26,371-375$.

Concione B., Musaccho O. : Contributo allo studio della trichinosi in Calabria. Alti Soc. Peloritana Sci. fis. mat. nat., 1966, 12, 381-385.

Corselli A., Romano R., Persiani A. : Ulteriori osservazioni sulla distribuzione della trichinellosi silvestre in volpi della Provincia di Roma. Parassitologia, 1983, 25, 249-251.

De Filippis V., Nardi E. : Un episodio di trichinosi umana verificatosi recentemente in provincia di Foggia. Parassitologia, 1968, 10, 219-221.

Istituto zooprofilattico deLl'abruzzo E DEL MOLISE : Relazione tecnica. 1982-1985.

Lo Nigro M. : Primo caso di trichinosi in Lucania. G. Mal. Infett. Parassit., 1958, 10, 414-416.

Lo Nigho M., Colella G., Lascaro M., Padula A., Annunziata L., Morelli A. : Un nuovo episodio di trichinosi umana in Basilicata da consumo di carne di cinghiale. Parassitologia, $1979,21,114-115$.

Mantovani A., Filippini I., Bergomi S. : Indagini su un'epidemia di trichinellosi umana verificatasi in Italia. Parassitologia, 1980, 22, 107-134.

Nardi E. : La trichinosi nei cani randagi del Grargano. Vet. Ital., 1960, 11, 532-536.

Orlandi V. : Indagini sulla trichinosi in provincia di Ascoli Piceno. Atti Soc. ital. Sci. vet., 1972, $26,482-484$.

Ozenetskovskaya N. N. : Pathogenesis, pathomorphology and clinics of trichinellosis: treatment of trichinellosis. In: Trichinelly i Trichinelliez (S. N. Boev, V. I. Bondareva, I. B. Sokolova, eds.), Akademija Nauk Kazahskoj, SSR, 1978, 165-213.

Pampiglione S., Doglioni L. : Osservazioni e ricerche su di un episodio epidemico di trichinosi verificatosi in provincia di Trento. Parassitologia, 1971, 13, 241-255.

Parravicini M., Grampa A., Salmini G., Parravicini V., Dietz A., Mon'tanari M. : Focolaio epidemico di trichinosi da carne di cavallo. G. Mal. Infett. Parassit., 1986, 38, 482-487.

Pozio E. : 'Trichinella from rats in Central Italy. J. Parasitol., 1986, $72,800-801$.

Pozio E., Rossi P., Amati M., Mancini Barbieri F. : Differenziazione genetica del genere Trichinella attraverso l'analisi isoenzimatica. Parassitologia, 1987, 29, (sous presse).

Sulzer A. J. : Indirect fluorescent antibody tests for parasitic diseases. 1. Preparation of the stable antigen from larvae of Trichinella spiralis. J. Parasitol., 1965, 51, 717-721. 